\title{
TRATAMENTO CIRÚRGICO DAS LESÕES OSTEOCONDRAIS DO JOELHO COM MOSAICOPLASTIA
}

\author{
SURGICAL TREATMENT OF OSTEOCHONDRAL LESIONS \\ OF THE KNEE WITH MOSAICPLASTY
}

\begin{abstract}
Ozório de Almeida Lira Neto', Carlos Eduardo da Silveira Franciozi ', Geraldo Sérgio de Mello Granata Júnior², Antonio Altenor Bessa de Queiroz ${ }^{3}$, Mario Carneiro Filho ${ }^{4}$, Ricardo Dizioli Navarro ${ }^{5}$
\end{abstract}

\section{RESUMO}

Objetivo: Avaliação dos resultados funcionais da técnica de mosaicoplastia em joelhos de pacientes com lesão osteocondral. Métodos: No período de agosto de 1999 a março de 2005, 27 pacientes foram submetidos à mosaicoplastia do joelho. Vinte e um eram do sexo masculino e seis, do feminino. A idade variou de 16 a 64 anos (média de 38,1 anos). Dezessete lesões eram do lado direito e 10, do esquerdo. Em relação à localização da lesão, quatro (15\%) situaram-se no côndilo femoral lateral, 18 (66,5\%) no côndilo femoral medial e $5(18,5 \%)$ na patela. O tamanho das lesões variou de 1 a $8 \mathrm{~cm}^{2}$ (média de $2,7 \mathrm{~cm}^{2}$ ). Os pacientes operados foram avaliados no pré e pós-operatórios pela escala funcional de Lysholm, com seguimento médio de 2,5 anos. Resultados: O pré-operatório teve uma média de 62,7 pontos e o pós-operatório uma média de 95,4 pontos. Os pacientes submetidos à mosaicoplastia no côndilo femoral lateral apresentaram, no pré-operatório, a média de pontos de 51,5 pontos e, no pósoperatório, média de 100 pontos. No côndilo femoral medial a média no pré-operatório foi de 64,1 pontos e, no pós-operatório, de 95,4 pontos. Com relação à patela, a média do pré-operatório foi de 66,4 pontos e, do pós-operatório, de 92 pontos. Conclusão: A mosaicoplastia mostrou-se uma boa alternativa no tratamento das lesões osteocondrais do joelho. Entretanto, apresentou melhor evolução nas lesões dos côndilos femorais em relação às localizadas na patela.

Descritores - Doenças das cartilagens/cirurgia; Cartilagem articular/transplante; Articulação do joelho/cirurgia; Artroscopia; Transplante autólogo

\section{ABSTRACT}

Objective: To evaluate the functional results of mosaicplasty in knees of patients with osteochondral lesion. Methods: Between August 1999 and March 2005, twenty-seven patients were submitted to mosaicplasty on the knee. Twenty-one were male and six female. The patients'ages ranged from 16 to 64 years (average 38.1 years). Seventeen lesions were located on the right knee and ten on the left one. The lesion was located on the lateral condyle in 4 patients (15\%), on the medial condyle in 18 patients (66.5\%) and on the patella in 5 patients (18.5\%). The lesion sizes varied from 1 to $8 \mathrm{~cm}^{2}$ (average $2.7 \mathrm{~cm}^{2}$ ). The patients operated on were evaluated before and after surgery by the functional Lysholm scale, with an average follow-up of 2.5 years. Results: In the preoperative evaluation, the average was 62.7 points, evolving to 95.4 points in the postoperative evaluation. The patients submitted to mosaicplasty in the lateral condyle presented an average of 51.5 points before surgery, evolving to 100 points after surgery. The patients submitted to mosaicplasty in the medial condyle had presented average of 64.1 points before surgery, evolving to 95.4 points after surgery. The patients submitted to mosaicplasty on the patella presented average of 66.4 points before surgery, evolving to 92 points in the postoperative evaluation. Conclusion: Mosaicplasty proved to be a good alternative for the treatment of chondral and osteochondral lesions of the knee, showing better evolution in lesions located on the femoral condyles than in lesions located on the patella.

Keywords - Cartilage diseases/surgery; Joint/transplantation cartilage; Knee joint/surgery; Arthroscopy; Autologous transplantation

\footnotetext{
1 - Médico do Grupo de Joelho da Disciplina de Ortopedia do DOT-Unifesp.

2 - Doutor em Ciências pela UNIFESP, Médico do Grupo do Joelho da Disciplina de Ortopedia do DOT - Unifesp.

3 - Mestre em Ciências pela Unifesp, Médico do Grupo de Joelho da Disciplina de Ortopedia do DOT- Unifesp.

4 - Professor Afiliado - Doutor em Ciências pela Unifesp.

5 - Professor Associado - Livre Docente, Chefe do Grupo de Joelho da Disciplina de Ortopedia do DOT- Unifesp.
}

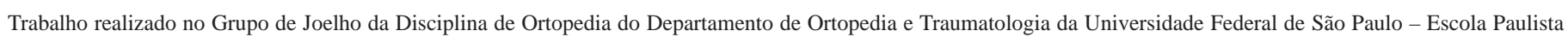
de Medicina.

Correspondência: Rua Visconde de Inhaúma, 81, apto. 94 -04145-030 - E-mail:cacarlos66@hotmail.com 


\section{INTRODUÇÃO}

As lesões condrais e osteocondrais no joelho são ainda um dos maiores problemas para o cirurgião de joelho, especialmente quando ocorrem em indivíduos jovens e atletas. A preservação da estrutura anatômica e das propriedades fisiológicas da cartilagem é um requisito básico para uma boa função da articulação.

A capacidade de regeneração cartilaginosa é limitada devido à incapacidade do condrócito de diferenciar-se e se multiplicar.

As lesões podem causar dor, edema e bloqueio articular e, se não tratadas adequadamente, causam a osteoartrose, principalmente se estiverem localizadas nas áreas de carga.

Existem vários tipos de tratamento cirúrgico para estas lesões, e são basicamente divididos em métodos reparativos, que estimulam e possibilitam a formação de tecido condrogênico ou fibrocondrogênico, e métodos reconstrutivos, que tratam o defeito com enxerto condral ou osteocondral autólogo ou homólogo ${ }^{(1)}$.

As técnicas reparativas como o desbridamento da lesão, a condroplastia por abrasão, as perfurações subcondrais e, mais recentemente, as microfraturas têm como objetivo a formação de um tecido fibrocartilaginoso com estrutura e biomecânica semelhantes, mas inferiores à cartilagem normal $^{(2)}$. No entanto, a longo prazo, não impedem a progressão da degeneração e, em um ano, as lesões já demonstram nova fibrilação e morte celular ${ }^{(3,4)}$.

Existem outros métodos alternativos como o enxerto periosteal, enxerto osteocondral sintético, enxerto osteocondral autólogo (mosaicoplastia) e o transplante de condrócitos ${ }^{(2)}$.

A técnica da mosaicoplastia descrita por Hangody em 1992 e publicada em 1997 demonstra, através de avaliações macroscópicas e microscópicas, a sobrevivência da cartilagem hialina transplantada com formação de um leito composto de $80 \%$ de cartilagem e $20 \%$ de fibrocartilagem na base do defeito ósseo, integração do enxerto com a matriz óssea do receptor e locais doadores preenchidos com osso esponjoso recoberto por uma capa de fibrocartilagem no final de oito semanas ${ }^{(5,6)}$.

A técnica consiste em retirar pequenos cilindros ósseos da periferia do côndilo femoral e da região intercondiliana, que são transplantados para as áreas em que existem lesões condrais e osteocondrais, com o objetivo de manter a qualidade e estrutura da cartilagem que compõem esta região ${ }^{(7)}$.

A literatura, segundo Hangody et $a l^{(5-8)}$, nos traz indicações e contraindicações para esse tipo de cirurgia (Quadro 1).
Quadro 1 - Indicações e contraindicações

\begin{tabular}{|c|c|c|}
\hline INDICAÇÕES & $\begin{array}{c}\text { CONTRAINDICAÇÕES } \\
\text { ABSOLUTAS }\end{array}$ & $\begin{array}{c}\text { CONTRAINDICAÇÕES } \\
\text { RELATIVAS }\end{array}$ \\
\hline $\begin{array}{l}\text { Lesões condrais e osteocondrais } \\
\text { focais na superfície articular em } \\
\text { área de carga }\end{array}$ & $\begin{array}{l}\text { Tumor, infecção, artrite } \\
\text { reumatóide, osteoartrose } \\
\text { avançada }\end{array}$ & Idade entre 40 e 50 anos \\
\hline Idade menor que 50 anos & $\begin{array}{l}\text { Falta de área doadora } \\
\text { disponível }\end{array}$ & Defeitos entre 4 e $8 \mathrm{~cm}^{2}$ \\
\hline $\begin{array}{l}\text { Área da lesão entre } 1 \text { e } 4 \mathrm{~cm}^{2} \text {, } \\
\text { podendo esta ser até } 8 \mathrm{~cm}^{2} \mathrm{em} \\
\text { casos isolados como procedi- } \\
\text { mento de salvação }\end{array}$ & Idade maior que 50 anos & Artrose intermediária \\
\hline $\begin{array}{l}\text { Tratamento concomitante ou } \\
\text { prévio de instabilidade, mau ali- } \\
\text { nhamento e lesões meniscais }\end{array}$ & Lesão maior que $8 \mathrm{~cm}^{2}$ & \\
\hline $\begin{array}{l}\text { Aceitação do paciente para co- } \\
\text { laboração na descarga de peso } \\
\text { pós-operatório }\end{array}$ & & \\
\hline
\end{tabular}

Este trabalho tem como objetivo avaliar funcionalmente os pacientes submetidos à técnica de mosaicoplastia para o tratamento das lesões osteocondrais do joelho.

\section{MÉTODOS}

No período entre agosto de 1999 e março de 2005, 27 pacientes com lesões osteocondrais no joelho foram submetidos à mosaicoplastia. A média de idade dos pacientes foi de 31,1 anos (16-64 anos). Dos 27 pacientes, 21 eram do sexo masculino e seis, do feminino. Dezessete pacientes foram operados do lado direito e 10, do esquerdo.

O tamanho médio do defeito foi de $2,7 \mathrm{~cm}^{2}\left(1-8 \mathrm{~cm}^{2}\right)$. Em relação ao local da lesão, quatro estavam localizadas no côndilo femoral lateral, 18 no medial e cinco, na patela (Tabela 1).

Os pacientes foram avaliados no pré e pós-operatórios pela escala funcional de Lysholm, com seguimento médio de 2,5 anos.

A escala de pontuação simplificada utilizada no questionário funcional de Lysholm consiste em: excelente - se o total de pontos estiver situado entre 95 e 100, bom - entre 84 e 94, regular - entre 65 e 83, e ruim - se menor que 64 .

Dos 27 pacientes, cinco tinham sido submetidos a cirurgias anteriores, sendo uma paciente submetida a três procedimentos: retirada da gordura de Hoffa, condroplastia e "release” lateral com "drilling” da patela e os outros quatro pacientes, submetidos respectivamente à retirada de corpo livre, “drilling” do côndilo femoral medial, meniscectomia parcial do menisco medial e meniscectomia parcial com exérese de cisto meniscal (Figura 1). 
Tabela 1 - Caracterização da amostra

\begin{tabular}{l|c}
\multicolumn{1}{c|}{ Variáveis } & $\mathrm{n}=27$ \\
\hline Idade (anos) - média (dp) & $38,1(13,1)$ \\
\hline mínimo - máximo & $16-64$ \\
\hline Sexo - $\mathrm{n}(\%)$ & $6(22,2)$ \\
\hline Feminino & $21(77,8)$ \\
\hline Masculino & \multicolumn{2}{|c}{} \\
\hline Lado operado $\mathrm{n}(\%)$ & $17(63,0)$ \\
\hline Direito & $10(37,0)$ \\
\hline Esquerdo & \\
\hline Local da lesão $n(\%)$ & $18(66,7)$ \\
\hline Côndilo femoral medial & $4(14,8)$ \\
\hline Côndilo femoral lateral & $5(18,5)$ \\
\hline Patela & $2,7(1,8)$ \\
\hline Tamanho da lesão (cm²) - média (dp) & $1-8$ \\
\hline mínimo - máximo & $2,3(1,5)$ \\
\hline Tempo pós-operatório (anos) - média (dp) & $0,3-6,0$ \\
\hline mínimo - máximo &
\end{tabular}

Fonte: Departamento de Ortopedia e Traumatologia da Universidade Federal de São Paulo

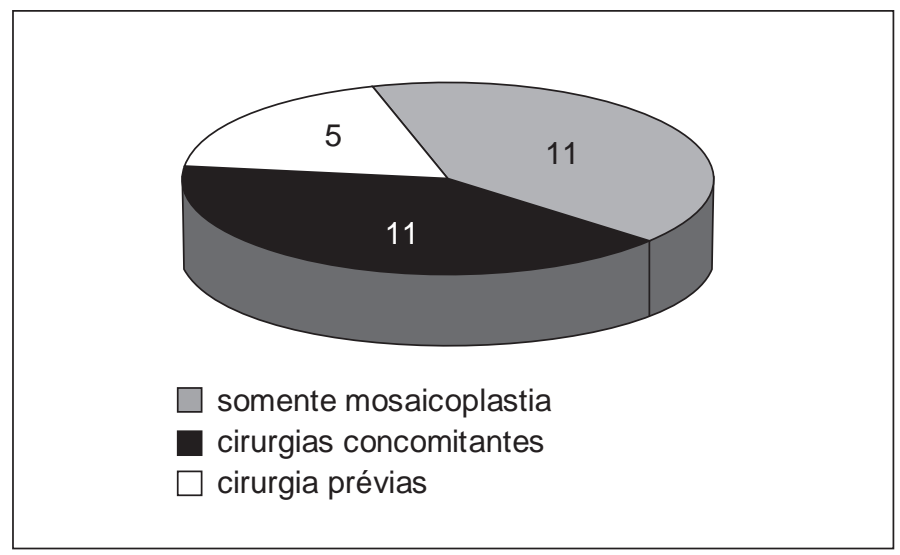

Fonte: Departamento de Ortopedia e Traumatologia da Universidade Federal de São Paulo

Figura 1 - Procedimentos

Onze pacientes foram tratados concomitantemente de outras lesões. Destas, temos quatro meniscectomias, três osteotomias valgizantes fixadas com placa de Puddu, duas reconstruções do ligamento cruzado anterior e duas reconstruções de ligamento cruzado anterior associadas à osteotomia valgizante fixadas com placa de Puddu (Figura 1).

A técnica da mosaicoplastia consiste em obter pequenos cilindros osteocondrais de uma área com mínima descarga de peso e transplantá-los para áreas defeituosas na região de carga. Utilizando-se de diferentes combinações de tamanhos de enxerto pode-se preencher de 90 a 100\% do defeito (Figura 2).

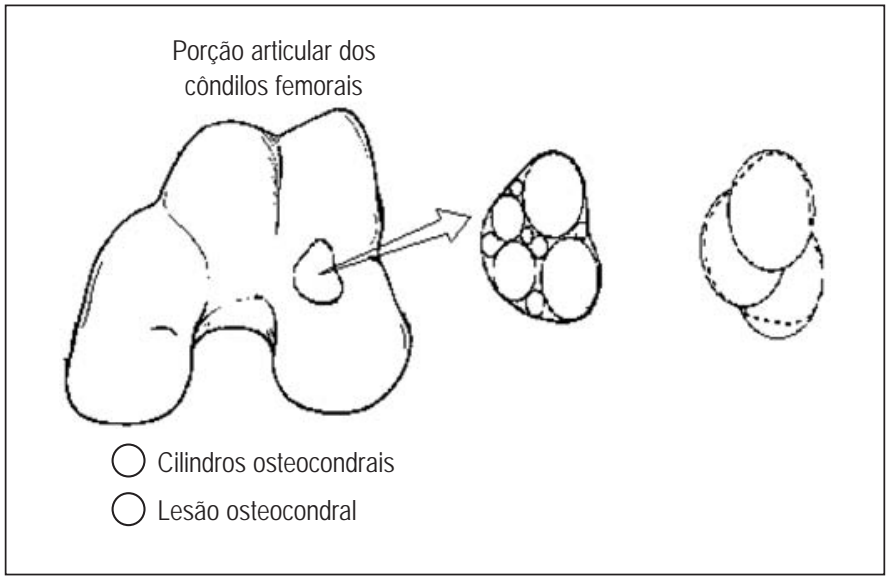

Fonte: Departamento de Ortopedia e Traumatologia da Universidade Federal de São Paulo

Figura 2 - Mosaicoplastia

A técnica pode ser feita por via aberta, miniartrotomia (Figura 3), ou via artroscópica (Figura 4), através de procedimentos semelhantes com apenas alguns passos realizados de maneira diferente ${ }^{(7)}$.

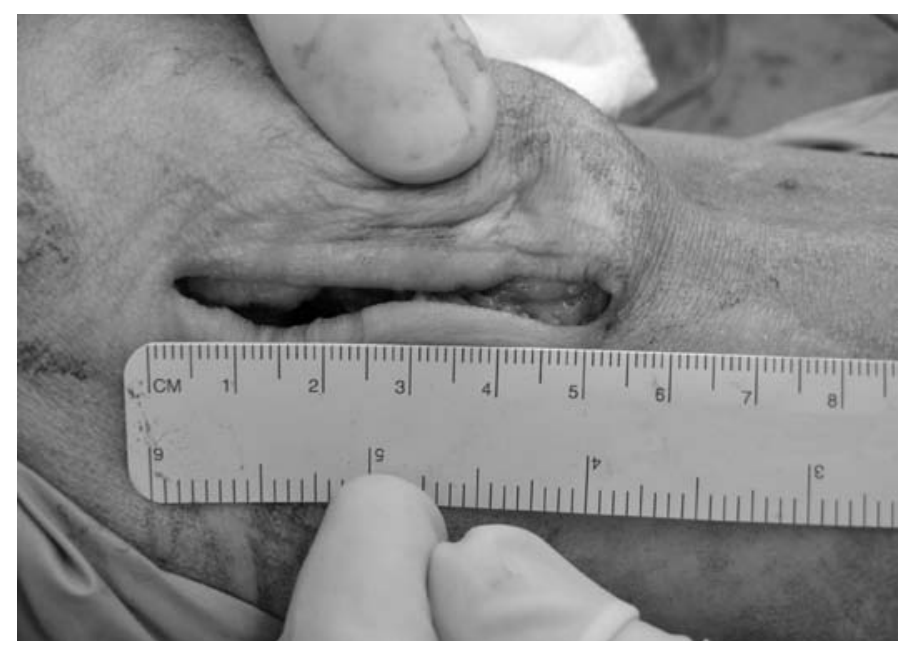

Figura 3 - Via aberta

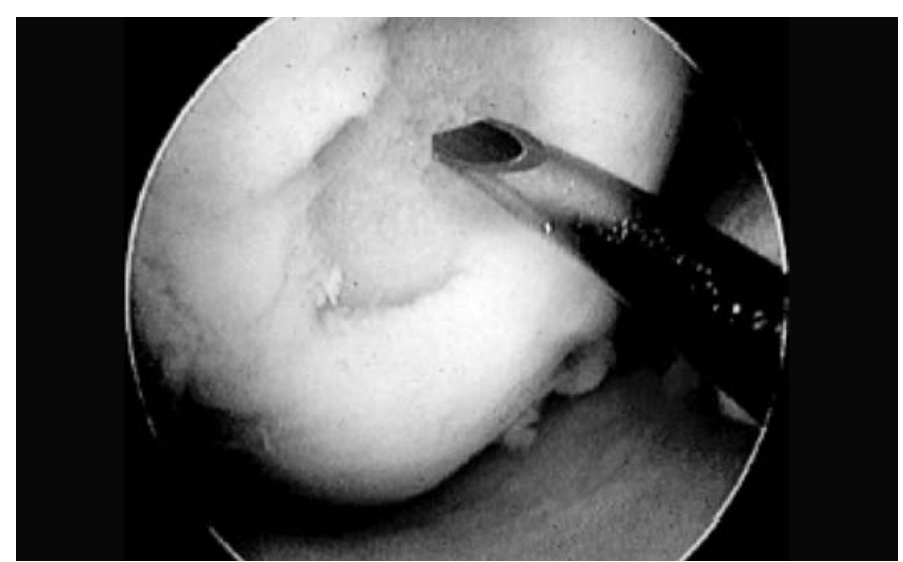

Fonte: Departamento de Ortopedia e Traumatologia da Universidade Federal de São Paulo

Figura 4 - Via artroscópica 
Das 22 lesões osteocondrais sem cirurgia prévia, seis foram abordadas pela técnica da miniartrotomia e 16, pela artroscopia. Cinco lesões pertenciam a pacientes que foram submetidos a cirurgias anteriores.

No procedimento artroscópico, a localização dos portais é de extrema importância. O paciente é colocado em posição supina, com o joelho livre para fletir até 120 graus. Utilizamos uma agulha para encontrar a melhor posição do portal para que, através dele, a lesão fosse alcançada de maneira perpendicular ao instrumental que será usado para retirada e colocação do cilindro osteocondral ${ }^{(7)}$.

Normalmente estes portais são mais mediais que os portais habituais. As lesões no côndilo femoral medial por osteocondrite dissecante, na sua localização mais habitual, são melhor abordadas pelo portal lateral. Por outro lado, um portal central trans-tendão patelar possibilita um bom acesso à região interna dos côndilos femorais medial e lateral.

Identificada a lesão, esta é desbridada até a exposição de osso subcondral viável em sua base. Com um guia de prova, o tamanho da lesão é medido e calculam-se quantos e qual tamanho dos cilindros serão utilizados (Figuras 5 e 6).

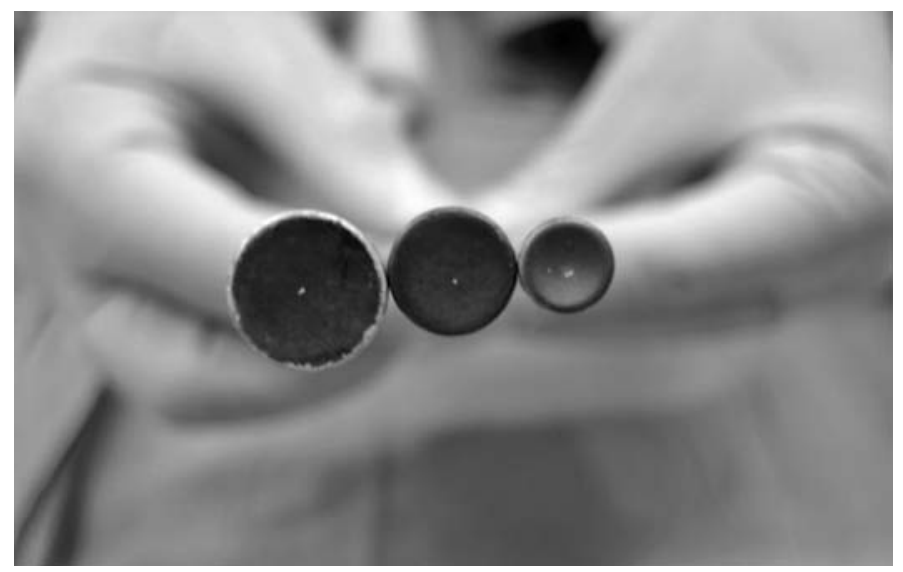

Figura 5 - Guias de prova

A seguir, com o joelho em extensão, abordamos a área doadora. No procedimento aberto, utiliza-se o côndilo femoral medial ou lateral. No procedimento artroscópico, utilizamos o côndilo femoral medial como primeira escolha, pois a patela se desloca lateralmente quando se insufla a articulação, conforme o descrito por Hangody et $a^{(7)}$. Nas lesões maiores foi necessário retirar o enxerto de ambos os côndilos.

Com um instrumento próprio para ressecção retiramos cilindros de diferentes diâmetros, todos com 15mm de extensão, na quantidade necessária para preencher a

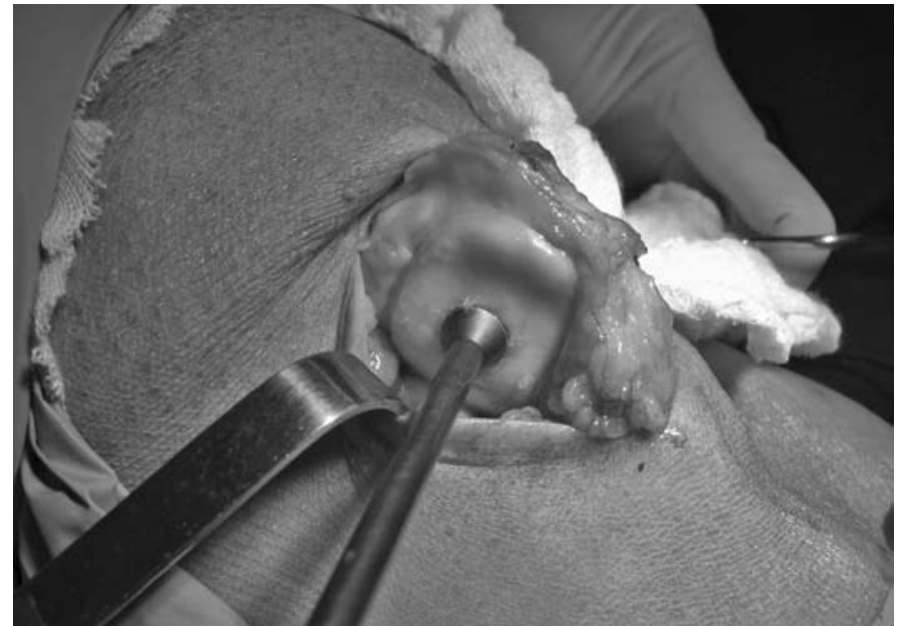

Fonte: Departamento de Ortopedia e Traumatologia da Universidade Federal de São Paulo

Figura 6 - Determinando o número de cilindros

lesão. Consideramos o sulco terminal como um limite anatômico inferior para a ressecção dos enxertos no côndilo femoral lateral (Figuras 7, 8 e 9).

Voltando à área receptora, com uma trefina, a lesão era perfurada até a profundidade desejada e então um dilatador do tamanho do enxerto era utilizado sob pressão, para regularizar o túnel, sempre perpendicular ao defeito existente. Com o instrumental apropriado o enxerto era colocado no defeito de maneira suave para evitar a lesão do cilindro osteocondral. Este procedimento de fresar, dilatar e colocar, era realizado quantas vezes fossem necessárias até o máximo preenchimento do local da lesão com cilindros osteocondrais (Figuras 10, 11 e 12).

Para as lesões da patela sempre fizemos procedimentos abertos. Através de artrotomia lateral, a patela era inclinada para visualizar a lesão realizando o procedimento descrito anteriormente. Nestes casos sempre utilizamos como área doadora o côndilo femoral lateral.

Independente da via, após a colocação dos cilindros osteocondrais, o joelho era fletido e estendido para verificar a excursão das estruturas na área enxertada com posterior fechamento por planos até a pele.

Utilizamos dreno em todos os pacientes, retirando-o após 24 horas do final da cirurgia.

Quanto à reabilitação, os pacientes eram liberados para arco de movimento passivo após a retirada do dreno no segundo pós-operatório, podendo fletir o joelho livremente conforme o tolerado, mantendo-o sem carga durante seis semanas. Nas duas semanas seguintes liberamos a carga parcial, atingindo a carga total com oito semanas. $\mathrm{O}$ retorno ao esporte ocorreu após quatro a seis meses.

Os valores obtidos foram submetidos à análise estatística, através do testes de Wilcoxon e de Mann-Whitney. 

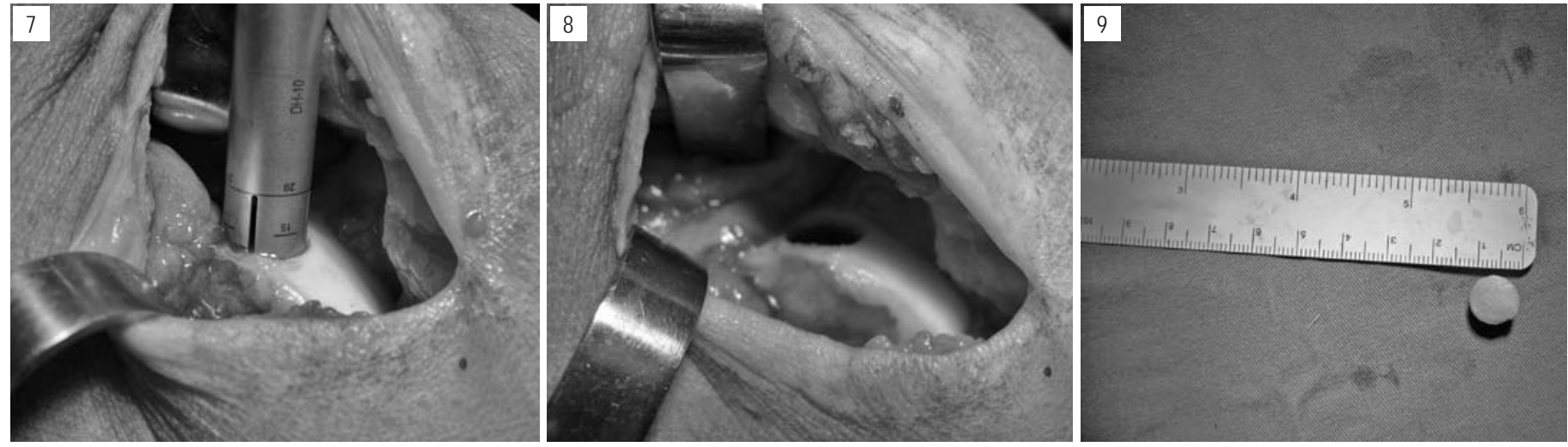

Fonte: Departamento de Ortopedia e Traumatologia da Universidade Federal de São Paulo

Figuras 7, 8 e 9 - Retirada do enxerto
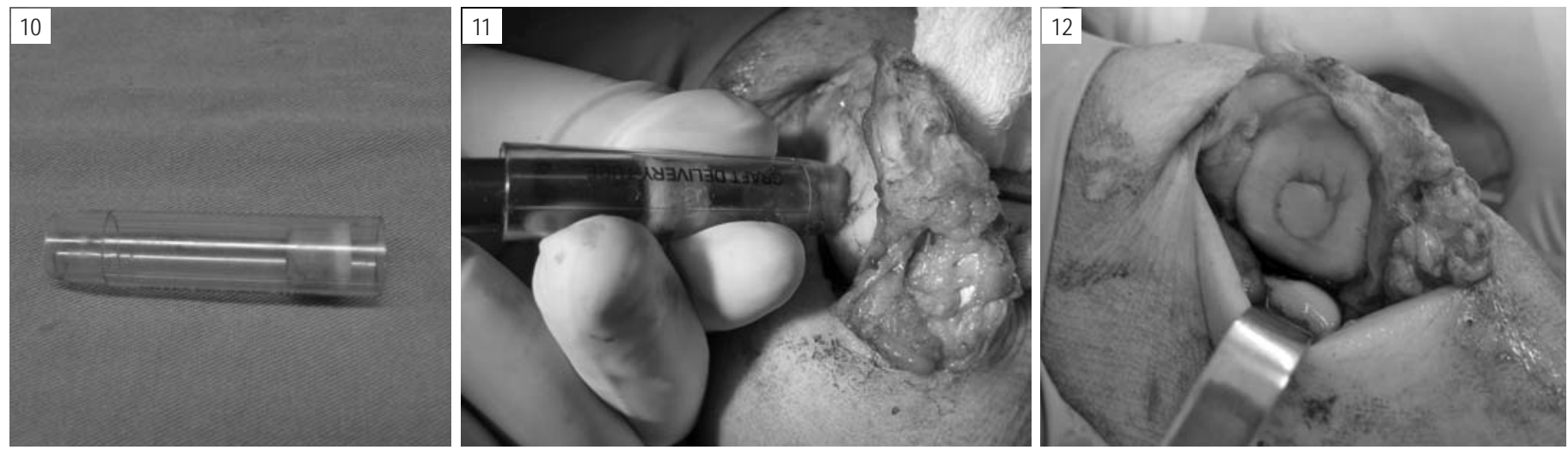

Fonte: Departamento de Ortopedia e Traumatologia da Universidade Federal de São Paulo

Figuras 10, 11 e 12 - Colocação do enxerto

\section{RESULTADOS}

Os pacientes foram submetidos à avaliação funcional pré e pós-operatória e classificados conforme o questionário de Lysholm, sendo seguidos por um tempo médio de 2,5 anos. Foi considerada a avaliação mais recente de cada paciente como resultado final definitivo.

O valor médio pré-operatório para os 27 pacientes foi de 63 pontos (ruim) e o valor médio pós-operatório foi de 95 pontos (excelente).

Na comparação entre as aplicações pré e pós-operatórias da escala Lysholm foi encontrada variação estatisticamente significante ( $\mathrm{p}<0,001$ ). Todos os pacientes apresentaram aumento da pontuação da escala na aplicação pós quando comparada à pré-operatória, com média de aumento de 59,9\% (dp $=40,6 \%$ ), variando entre $11,3 \%$ e $135,7 \%$ (Tabela 2 ).

Se avaliarmos os pacientes conforme o local da lesão - patela, côndilo femoral lateral (CFL) e côndilo femoral medial (CFM) -, os valores variam discretamente. Para as lesões na patela os pacientes apresentavam, no préoperatório, 66 pontos (regular) e, no pós-operatório, 92
Tabela 2 - Escala de Lysholm

\begin{tabular}{c|c|c|c|c|c}
\hline \multirow{2}{*}{ Aplicação } & \multicolumn{5}{c}{ Escala Lysholm (n=27) } \\
\cline { 2 - 6 } & Média & $\mathrm{dp}$ & Mediana & Mínimo & Máximo \\
\hline Pré-operatória & 62,7 & 12,8 & 65 & 42 & 83 \\
\hline Pós-operatória & 95,4 & 6,7 & 99 & 78 & 100 \\
\hline$\Delta \%$ & 59,9 & 40,6 & 47,7 & 11,3 & 135,7 \\
\hline
\end{tabular}

pontos (bom). Já os pacientes com lesão no côndilo femoral lateral tinham, no pré-operatório, 51 pontos (ruim) e, no pós-operatório, 100 pontos (excelente). No que diz respeito aos pacientes com lesão no côndilo femoral medial, o valor pré-operatório era de 66 pontos (regular) evoluindo para 95 pontos no pós-operatório (excelente).

Analisando estes valores quanto ao local, não foi encontrada diferença estatisticamente significante entre os grupos quanto à pontuação da escala Lysholm nas aplicações pré-operatória ( $\mathrm{p}=0,147)$, pós-operatória ( $\mathrm{p}$ $=0,060)$ e variação $\Delta \%(\mathrm{p}=0,125)$ (Tabela 3). 
Tabela 3 - Escala de Lysholm segundo o local da lesão

\begin{tabular}{l|c|c|c}
\hline \multirow{2}{*}{ Local da Lesão } & \multicolumn{3}{|c}{ Escala Lysholm } \\
\cline { 2 - 4 } & Pré-operatória & Pós-operatória & $\%$ \\
\hline Côndilo femoral medial & $64,1(11,9)$ & $95,4(6,4)$ & $54,7(35,4)$ \\
$n=18$ & $42-83$ & $79-100$ & $11,3-126,2$ \\
\hline Côndilo femoral lateral & $51,5(11,0)$ & $100,0(0,0)$ & $99,9(35,4)$ \\
$n=4$ & $45-68$ & $100-100$ & $47,1-122,2$ \\
\hline Patela ( $n=5)$ & $66,4(14,7)$ & $92,0(8,8)$ & $46,6(49,9)$ \\
\hline Prova de Mann-Whitney & $42-80$ & $78-99$ & $20,0-135,7$ \\
\hline Valores expressos emm & $p=0,147$ & $P=0,060$ & $p=0,125$ \\
\hline
\end{tabular}

Valores expressos em média (dp), mínimo - máximo

Fonte: Departamento de Ortopedia e Traumatologia da Universidade Federal de São Paulo

Dividindo-se a amostra segundo o tamanho da lesão, não foi encontrada diferença estatisticamente significante entre os grupos quanto à pontuação da escala Lysholm nas aplicações pré-operatória $(\mathrm{p}=0,693)$, pós-operatória $(\mathrm{p}=0,066)$ e variação $\Delta \%(\mathrm{p}=0,657)$ (Tabela 4).

Tabela 4 - Escala de Lysholm segundo o tamanho da lesão

\begin{tabular}{l|c|c|c}
\hline \multirow{2}{*}{ Tamanho da lesão } & \multicolumn{3}{|c}{ Escala Lysholm } \\
\cline { 2 - 4 } & Pré-operatória & Pós-operatória & $\%$ \\
\hline \multirow{2}{*}{$\leq 2 \mathrm{~cm}^{2}(\mathrm{n}=16)$} & $62,0(12,0)$ & $93,3(7,8)$ & $57,8(42,6)$ \\
& $42-80$ & $78-100$ & $11,3-135,7$ \\
\hline \multirow{2}{*}{$2 \mathrm{~cm}^{2}(\mathrm{n}=11)$} & $63,6(14,3)$ & $98,6(2,2)$ & $62,9(39,2)$ \\
& $46-83$ & $95-100$ & $17,3-117,4$ \\
\hline Prova de Mann-Whitney & $\mathrm{p}=0,693$ & $\mathrm{p}=0,066$ & $\mathrm{p}=0,657$ \\
\hline Valores expressos em média $(\mathrm{dp})$ mínimo - máximo
\end{tabular}

Fonte: Departamento de Ortopedia e Traumatologia da Universidade Federal de São Paulo

Analisando conforme a idade, pacientes com menos de 40 anos apresentavam 64 pontos no pré-operatório e 94 no pós-operatório, e pacientes com 40 anos ou mais apresentaram 60 pontos no pré-operatório e 92 pontos no pós-operatório. Não foi encontrada diferença estatisticamente significante entre os grupos etários quanto à pontuação da escala Lysholm nas aplicações pré-operatória $(\mathrm{p}=0,464)$, pós-operatória $(\mathrm{p}=0,392)$ e variação $\Delta \%(\mathrm{p}=0,367)$ (Tabela 5).

Tabela 5 - Escala de Lysholm segundo a idade do paciente

\begin{tabular}{l|c|c|c}
\hline \multirow{2}{*}{\multicolumn{1}{c}{ Idade }} & \multicolumn{3}{|c}{ Escala Lysholm } \\
\cline { 2 - 4 } & Pré-operatória & Pós-operatória & $\Delta \%$ \\
\hline \multirow{2}{*}{$<40$ anos $(n=15)$} & $64,1(13,4)$ & $93,8(8,2)$ & $54,4(43,7)$ \\
& $42-83$ & $78-100$ & $13,7-126,2$ \\
\hline \multirow{2}{*}{$\geq 40$ anos ( $n=12)$} & $60,9(12,2)$ & $97,5(3,4)$ & $66,8(36,9)$ \\
& $42-81$ & $89-100$ & $11,3-135,7$ \\
\hline Prova de Mann-Whitney & $p=0,464$ & $p=0,392$ & $p=0,367$ \\
\hline Valores expressos em média (dp), mínimo - máximo
\end{tabular}

Fonte: Departamento de Ortopedia e Traumatologia da Universidade Federal de São Paulo
Por fim, dividimos os pacientes em três grupos: um que só realizou mosaicoplastia (grupo 1), outro que realizou mosaicoplastia com outras cirurgias concomitantes (grupo 2), e um terceiro grupo de pacientes que já tinham realizado cirurgias prévias à mosaicoplastia (grupo 3). Dessa maneira, obtivemos o seguinte resultado: o grupo 1 apresentou média de 62 pontos no pré-operatório, passando para 97 pontos no pós-operatório. O grupo 2, com 59 pontos no pré-operatório, apresentou 95 pontos no pós-operatório e o grupo 3, com 71 pontos no préoperatório, apresentou 91 pontos no pós-operatório. Não houve diferença estatisticamente significante entre os três grupos (Figura 13).

\section{Lysholm X Procedimentos}
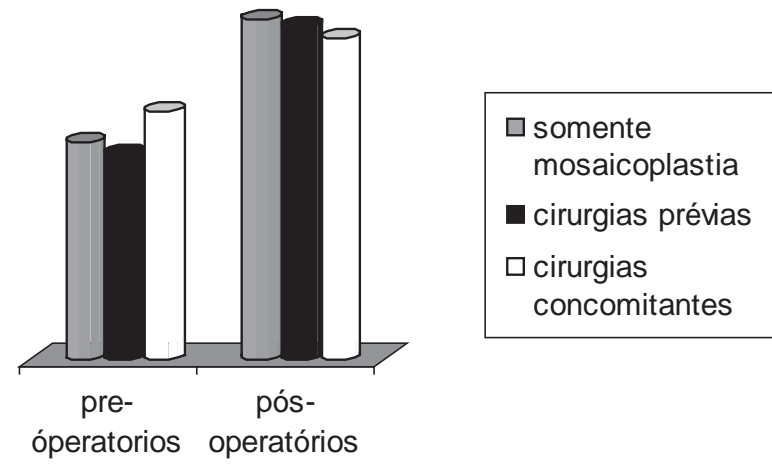

óperatorios operatórios

Fonte: Departamento de Ortopedia e Traumatologia da Universidade Federal de São Paulo

Figura 13 - Procedimentos

No que diz respeito a complicações, dois pacientes apresentaram quadro de infecção no pós-operatório imediato. Ambos os pacientes foram submetidos à osteotomia valgizante com a técnica da placa de Puddu. Um paciente apresentou uma infecção superficial e foi tratado com antibioticoterapia e limpeza local. O outro, com infecção profunda no local da osteotomia, foi tratado com retirada do enxerto liofilizado, limpeza cirúrgica e antibioticoterapia. Ambos os pacientes evoluíram bem com a cura da infecção.

\section{DISCUSSÃO}

A técnica da mosaicoplastia foi escolhida para o tratamento das lesões de cartilagem com base nos achados da literatura. Um estudo multicêntrico randomizado realizado por Hangody et al $^{(9)}$ comparou quatro técnicas diferentes para tratamento de lesões de cartilagem 
(“drilling”, artroplastia de abrasão, microfratura e mosaicoplastia) e mostrou uma melhora substancial da clínica e função dos pacientes submetidos a mosaicoplastia em relação às outras técnicas reparativas, especialmente após três, quatro e cinco anos.

Quando comparada à técnica reconstrutiva como transplante de condrócitos, Dozin et al ${ }^{(10)}$, em um estudo randomizado multicêntrico, concluíram que as duas técnicas são funcionalmente e clinicamente semelhantes, com recuperação total em $85 \%$ e $68 \%$ dos pacientes submetidos a mosaicoplastia e transplante de condrócitos, respectivamente. Já Bentley et $a l^{(11)}$, em um estudo randomizado prospectivo com 100 pacientes, obtiveram $78 \%$ e $69 \%$ de bons resultados em pacientes submetidos a transplante de condrócitos e mosaicoplastia, respectivamente.

Utilizamos a mosaicoplastia por acreditarmos que esta técnica é superior às técnicas reparativas já citadas e que apresenta resultado semelhante ao transplante de condrócitos, que tem como principal dificuldade o difícil acesso e o alto custo do procedimento.

O seguimento do estudo (2,5 anos) não é longo o suficiente para avaliarmos definitivamente a durabilidade dos resultados e a sobrevivência dos enxertos, mas os resultados mostram que é uma técnica segura e eficaz a curto e médio prazos.

Os resultados do trabalho, baseados na avaliação de Lysholm, mostraram uma melhora estatisticamente significante da função dos pacientes com média de 62,7 pontos (ruim) para 95,4 pontos (excelente).

Estes resultados vão ao encontro da literatura segundo vários autores como Marcacci et $a^{(1)}$ que apresentam 78,3\% de bons resultados, Jakob et al ${ }^{(2)}$ com 100\% de melhora da função e com 93\% de bons resultados, Hangody et al $^{(7)}$ com aproximadamente $90 \%$ de bons resultados em 831 procedimentos e Koulalis et al ${ }^{(12)}$ com $90 \%$ de bons resultados em três a seis meses após a cirurgia.

No que diz respeito à via de acesso, Hangody et $a l^{(5)}$, no seu primeiro relato da técnica, preconizavam a via artroscópica apenas para as lesões de até $2 \mathrm{~cm}^{2} \mathrm{com}$, no máximo, quatro a seis cilindros osteocondrais a serem enxertados. Nas lesões maiores, indicavam a via aberta (miniartrotomia). Em publicações mais recentes, indicam a cirurgia aberta apenas em casos em que a área da lesão é de difícil acesso devido ao seu local ou à limitação da flexão do joelho.

A literatura apresenta trabalhos com bons resultados em que só foi utilizada a via aberta, como Jakob et $a l^{(2)}$, ou só via artroscópica como Marcacci et $a l^{(1)}$. Nós utilizamos a via artroscópica ou a miniartrotomia nas lesões condilares, enquanto que, nas lesões na patela, utilizamos sempre miniartrotomia lateral.

Os resultados foram divididos em dois grupos conforme a idade dos pacientes: um menor que 40 anos e outro de 40 anos ou mais, apesar de Hangody et al $^{(7)}$ preconizarem o tratamento de indivíduos de até 40 anos, considerando contraindicação relativa entre 40 e 50 anos e contraindicando o tratamento em pacientes maiores de 50 anos. Não encontramos diferença estatística nos resultados entre os dois grupos avaliados, apesar de a literatura mostrar melhores resultados em pacientes mais jovens ${ }^{(1,7,8)}$.

No que diz respeito ao tamanho da lesão, Hangody et $a^{(7)}$ indicam o procedimento preferencialmente em lesões entre 1 e $4 \mathrm{~cm}^{2}$, podendo em alguns casos de salvação chegar a até $8 \mathrm{~cm}^{2}$. No nosso estudo realizamos procedimentos em lesões entre 1 e $8 \mathrm{~cm}^{2}$ e, para avaliarmos os resultados, dividimos as lesões em até $2 \mathrm{~cm}^{2}$ e maiores que $2 \mathrm{~cm}^{2}$. Não obtivemos diferença estatisticamente significante entre os dois grupos, porém houve uma tendência a melhores resultados para lesões maiores que $2 \mathrm{~cm}^{2}$. Neste aspecto, a literatura é indefinida, pois autores como Marcacci et al ${ }^{(1)}$ relatam que, quanto menor a lesão, melhor o resultado e Hangody et $a l^{(7)}$ têm melhores resultados em lesões entre 1 e $4 \mathrm{~cm}^{2}$. Outros autores, como Jakob et al ${ }^{(2)}$, referem não haver diferença nos resultados com relação ao tamanho da lesão.

Quanto ao local da lesão, nossos resultados não mostraram diferença estatisticamente significante, porém tenderam para melhor nas lesões condilares em relação às lesões na patela. As lesões condilares localizadas no côndilo lateral evoluíram melhor que as do côndilo medial, confirmando os registros da literatura ${ }^{(1,6)}$.

Analisamos ainda os resultados dividindo a amostra em três grupos: um de pacientes apenas submetidos à mosaicoplastia, outro de pacientes submetidos à mosaicoplastia e cirurgias concomitantes num mesmo tempo, e um terceiro grupo de pacientes submetidos à mosaicoplastia mas que já haviam sido submetidos a procedimentos anteriores no mesmo joelho. Não encontramos diferença estatisticamente significante entre os três grupos, mas observamos melhores resultados em pacientes submetidos apenas à mosaicoplastia. Houve melhora significativa em pacientes submetidos a cirurgias concomitantes e piores resultados em pacientes submetidos a cirurgias anteriores. Estas tendências nos resultados 
concordam com a literatura em que Jakob et $a^{(2)}$ relatam melhores resultados em pacientes submetidos apenas à mosaicoplastia. Marcacci et $a^{(1)}$ também referem uma melhora funcional mais acentuada em pacientes submetidos a procedimentos concomitantes em relação a pacientes submetidos apenas à mosaicoplastia, porém piores resultados aconteceram no grupo de pacientes submetidos às cirurgias anteriores.

Acreditamos que a melhora da função dos pacientes submetidos às cirurgias concomitantes deve-se não só à mosaicoplastia, mas também ao tratamento das lesões associadas. Já os piores resultados relacionados às cirurgias anteriores podem ser pelo fato de que nestes casos as lesões são mais graves e algumas vezes já foram tratadas sem sucesso, o que pode comprometer a articulação de outras maneiras. Além disso, pode haver um possível ceticismo do paciente à obtenção de um bom resultado, o que alteraria a sua avaliação subjetiva na escala funcional.

Quanto à reabilitação, baseamos o protocolo na experiência pessoal com outros procedimentos para tratamento de lesão de cartilagem, juntamente com protocolos de tratamento para lesões concomitantes. Comparando com a literatura, observamos que os mesmos são bastante parecidos. Hangody et $a l^{(8)}$ preconizam movimentação passiva total sem carga por três semanas seguida de carga parcial por mais duas a três semanas. Jakob et $a l^{(2)}$ orientam a movimentação passiva até $100^{\circ}$ até a terceira semana e total a partir desta, com carga parcial por quatro a oito semanas, dependendo do tamanho da lesão, e com retorno ao esporte ou trabalho pesado em quatro a seis meses. Concordamos com a mobilização passiva precoce, mas preferimos manter o paciente sem carga por seis semanas, por acharmos que a carga parcial precoce é difícil de ser estabelecida e controlada pelo paciente. Desta forma, eliminamos o risco da carga total antes do momento adequado.

\section{CONCLUSÃO}

A mosaicoplastia mostrou-se uma boa alternativa no tratamento das lesões osteocondrais do joelho. A idade até 65 anos e o tamanho da lesão não são fatores limitantes. Houve melhor evolução das lesões dos côndilos femorais em relação às localizadas na patela embora não tenhamos encontrado diferença estatisticamente significante neste aspecto. A realização de cirurgias associadas ou cirurgias prévias não inviabiliza o procedimento por nós estudado.

\section{AGRADECIMENTOS}

Agradecemos ao Dr. Marcel Jun Sugawara Tamaoki, sem o qual não teria sido possível a realização desse trabalho.

\section{REFERÊNCIAS}

1. Marcacci M, Kon E, Zaffagnini S, lacono F, Neri MP, Vascellari A, et al. Multiple osteochondral arthroscopic grafting (mosaicplasty) for cartilage defects of the knee: prospective study results at 2-year follow-up. Arthroscopy. 2005;21(4):462-70.

2. Jakob RP, Franz T, Gautier E, Mainil-Varlet P. Autologous osteochondral grafting in the knee: indication, results, and reflections. Clin Orthop Relat Res. 2002;(401):170-84.

3. Buckwalter JA. Chondral and osteochondral injuries. Mecanism of injury and repair responses. Oper Tech Orthop. 1997;7(4):263-9.

4. Suh JK, Aroen A, Muzzonigro TS, Disilvestro M, Fu FH. Injury and repair of articular cartilage. Operative Techniques in Orthopaedics 1997;7(4):270-8.

5. Hangody L, Kish G, Kárpáti Z, Szerb I, Udvarhelyi I. Arthroscopic autogenous osteochondral mosaicplasty for the treatment of femoral condylar articular defects. A preliminary report. Knee Surg Sports Traumatol Arthrosc. 1997;5(4):262-7.

6. Hangody L, Feczkó P, Bartha L, Bodó G, Kish G. Mosaicplasty for the treatment of articular defects of the knee and ankle. Clin Orthop Relat Res. 2001;(391 Suppl):S328-36.

7. Hangody L, Ráthonyi GK, Duska Z, Vásárhelyi G, Füles P, Módis L. Autolo-

gous osteochondral mosaicplasty. Surgical technique. J Bone Joint Surg Am. 2004;86(Suppl 1):65-72.

8. Hangody L, Füles P. Autologous osteochondral mosaicplasty for the treatment of full-thickness defects of weight-bearing joints: ten years of experimental and clinical experience. J Bone Joint Surg Am. 2003;85-A(Suppl 2):25-32.

9. Hangody L, Kish G, Kárpáti Z. Arthroscopic autogenous osteochondral mosaicplasty - a multicentric, comparative, prospective study. Index Traumatol Sport. 1998;5(1):3-9.

10. Dozin B, Malpeli M, Cancedda R, Bruzzi P, Calcagno S, Molfetta L, et al. Comparative evaluation of autologous chondrocyte implantation and mosaicplasty: a multicentered randomized clinical trial. Clin J Sport Med. 2005;15(4):220-6.

11. Bentley G, Biant LC, Carrington RW, Akmal M, Goldberg A, Williams AM, Skinner $\mathrm{JA}$, et al. A prospective, randomised comparison of autologous chondrocyte implantation versus mosaicplasty for osteochondral defects in the knee. J Bone Joint Surg Br. 2003;85(2):223-30.

12. Koulalis D, Schultz W, Heyden M, König F. Autologous osteochondral grafts in the treatment of cartilage defects of the knee joint. Knee Surg Sports Traumatol Arthrosc. 2004;12(4):329-34. 